\title{
Reflectance anisotropy spectroscopy of magnetite (110) surfaces
}

\author{
K. Fleischer, ${ }^{1, *}$ R. Verre, ${ }^{1,2}$ O. Mauit, ${ }^{3}$ R. G. S. Sofin, ${ }^{4}$ L. Farrell, ${ }^{3}$ C. Byrne,,${ }^{1}$ C. M. Smith, ${ }^{1}$ J. F. McGilp,,${ }^{1}$ and I. V. Shvets ${ }^{1,3}$ \\ ${ }^{1}$ School of Physics, Trinity College Dublin, Dublin 2, Ireland \\ ${ }^{2}$ Department of Applied Physics, Chalmers University of Technology, 41296 Göteborg, Sweden \\ ${ }^{3}$ Centre for Research on Adaptive Nanostructures and Nanodevices (CRANN), Trinity College Dublin, Dublin 2, Ireland \\ ${ }^{4}$ Department of Physics, College of Science Sultan Qaboos University, Muscat, Oman \\ (Received 13 August 2013; revised manuscript received 22 April 2014; published 13 May 2014)
}

\begin{abstract}
Reflectance anisotropy spectroscopy (RAS) has been used to measure the optical anisotropies of bulk and thin-film $\mathrm{Fe}_{3} \mathrm{O}_{4}(110)$ surfaces. The spectra indicate that small shifts in energy of the optical transitions, associated with anisotropic strain or electric field gradients caused by the (110) surface termination or a native oxide layer, are responsible for the strong signal observed. The RAS response was then measured as a function of temperature. A distinct change in the RAS line-shape amplitude was observed in the spectral range from 0.8 to $1.6 \mathrm{eV}$ for temperatures below the Verwey transition of the crystal. Finally, thin-film magnetite was grown by molecular beam epitaxy on $\mathrm{MgO}(110)$ substrates. Changes in the RAS spectra were found for different film thickness, suggesting that RAS can be used to monitor the growth of magnetite (110) films in situ. The thickness dependence of the RAS is discussed in terms of various models for the origin of the RAS signal.
\end{abstract}

DOI: 10.1103/PhysRevB.89.195118

PACS number(s): 78.20.-e, 71.27.+a, 71.30.+h, 42.65.Hw

\section{INTRODUCTION}

Magnetite $\left(\mathrm{Fe}_{3} \mathrm{O}_{4}\right)$ is the oldest known ferromagnetic material and it has been widely used, e.g., for compasses, magnetic data storage [1], and as a constituent of ferrofluids [2]. For these reasons, its properties have been widely investigated at different temperatures, from electrical $[3,4]$, magnetic [5,6], optical [7-11], and structural [12,13] points of view. Recently, there has been a renewed interest in magnetite: it is a ferromagnet with Curie temperature well above room temperature $(838 \mathrm{~K})$, it is stable in atmosphere, and has a half-metallic character. Magnetite crystallizes at room temperature (RT) in a cubic spinel structure. At $\sim 120$ $\mathrm{K}$ (Verwey temperature), a change in its structural properties takes place, resulting in a lowering of the symmetry of the system $[14,15]$. Together with the change in structure, a drop in the conductivity by two orders of magnitude [16] and changes in the far-infrared optical reflectivity [7] are also observed. This phenomenon, known as the Verwey transition, has been explained as an electronic order-disorder transition of the $\mathrm{Fe}$ ions occupying the octahedral B sites. Since its discovery, there has been great interest in this phenomenon, but its exact mechanism is still actively discussed [17-20]. A recent lowtemperature high-energy $\mathrm{x}$-ray diffraction study was able to determine the atomic positions and displacement vectors at the low-temperature state [19], while with Raman spectroscopy Yazdi et al. found indications that charge ordering occurs separately, but dependent on the structural changes [20]. Below the Verwey temperature, the minority electron band responsible for conduction is $\frac{1}{3}$ filled, forming a full spinpolarized half-metallic state which could be potentially used in spintronic applications.

For many practical applications [21] of magnetite inplane devices are required, but controlled stoichiometry [22] and crystallinity [23] of films is still challenging. In order

*fleisck@tcd.ie to improve control in the growth of this oxide, optical methods could be utilized in situ, without exposing the sample to the atmosphere, unlike ex situ methods such as $\mathrm{x}$-ray diffraction. Among the different techniques, reflectance anisotropy spectroscopy (RAS) readily presents itself as an ideal candidate due to its sensitivity and ease of use. However, the technique can only be applied to samples with in-plane surface anisotropies [24,25]. Nevertheless, a wide range of metal and semiconductor anisotropic crystalline surfaces have been previously studied by RAS [24-30]. In contrast, only a few reports on optical anisotropies of metal-oxide surfaces exist, mostly focused on wide-band-gap materials such as $\mathrm{ZnO}$ [31] or superconducting cuprates [32].

The optical properties of magnetite and their origin have been extensively discussed in previous studies, focused on the magneto-optical Kerr effect (MOKE) of the material and on the dielectric function based on ellipsometric or reflectance measurement [7,8,10,11,33-35]. In contrast, we present reflectance anisotropy spectra of the $\mathrm{Fe}_{3} \mathrm{O}_{4}(110)$ surface for bulk and thin-film samples. The measured RAS response is discussed in terms of changes in the electronic structures due the surface-induced strain or electric field gradients. The RAS of the sample has been investigated at different temperatures, and pronounced RAS changes were observed across the Verwey transition. We will show that RAS is significantly more sensitive to changes in the $\mathrm{Fe}_{3} \mathrm{O}_{4}$ electronic structure than traditional reflectance measurements.

First, we discuss the RAS of bulk $\mathrm{Fe}_{3} \mathrm{O}_{4}(110)$ and its magneto-optical Kerr rotation. The latter is required as the instrumental setup measures rotations in polarization upon normal incidence reflection, whether they are caused by reflectance anisotropy or the polar MOKE. By employing symmetry operations, both quantities can be distinguished [36,37].

Finally, we will discuss the differences in the reflectance anisotropy of thin-film $\mathrm{Fe}_{3} \mathrm{O}_{4}(110)$ grown on nearly latticematched $\mathrm{MgO}(110)$ with respect to bulk $\mathrm{Fe}_{3} \mathrm{O}_{4}(110)$, and how RAS can be employed as an in situ probe of film thickness. 


\section{EXPERIMENTAL METHODS}

For reflectance-based optical measurements, the bulk magnetite $\mathrm{Fe}_{3} \mathrm{O}_{4}(110)$ sample must be polished. Float zone grown synthetic crystals (Moscow State Steel and Alloys Institute) were glued on to a holder and initially polished using P2500 grade sand paper. Subsequently, diamond and cerium oxide suspensions with a final grain size of $0.01 \mu \mathrm{m}$ have been used for further polishing. During the polishing process, the sample was moved in a figure of 8 pattern and rotated occasionally in order to ensure that the polishing was smooth and did not cause any preferential direction which might influence any measurement of the samples anisotropy.

The magnetite thin films were grown on a $\mathrm{MgO}$ (110) substrate using oxygen plasma assisted molecular beam epitaxy (MBE) (base pressure $1.4 \times 10^{-9} \mathrm{mbar}$ ). Details of the growth conditions can be found elsewhere [38]. During the deposition, the substrate was maintained at a temperature of $520 \mathrm{~K}$ and $\mathrm{Fe}$ was evaporated from an $e$-beam evaporator. Reactive oxygen was supplied by an electron cyclotron resonance plasma source. The plasma source was operated at a power of $80 \mathrm{~W}$ at an oxygen partial pressure of $1.7 \times 10^{-5}$ mbar.

All thin-film samples have been characterized by $\mathrm{x}$-ray diffraction (XRD) and x-ray reflection (XRR) using a Bruker D8 Discover, with a monochromatic copper $K \alpha 1$ source and a position-sensitive detector (LynxEye) for reciprocal space maps and strain analysis.

RAS spectra were recorded at near-normal incidence with an in-house built reflectance anisotropy spectrometer which follows the Aspnes design [26]. Light from a Xe lamp passes through a Rochon $\mathrm{Mg}_{2} \mathrm{~F}$ polarizer and is reflected from the sample. The beam passes through a photoelastic modulator (PEM), an analyzing polarizer, and a monochromator before finally reaching a diode detector system. All measurements were performed from 0.35 to $5 \mathrm{eV}$, the broad spectral range has been realized using a Bentham TMc300 triple grating monochromator and three individual photo diodes (InAs, InGaAs, Si).

Spectroscopic ellipsometry of selected thin-film $\mathrm{Fe}_{3} \mathrm{O}_{4}$ samples have been performed on a Sopra GESP 5 spectroscopic ellipsometer using incidence angles of $69^{\circ}, 72^{\circ}$, and $75^{\circ}$ in an energy range from 1.5 to $5 \mathrm{~V}$. The thin-film dielectric function was extracted from the raw measurements by using the known film thickness and roughness from XRR.

For RT measurements, the sample was placed in an electromagnet, enabling MOKE measurements in a magnetic field of up to $250 \mathrm{mT}$. The magnetic field was calibrated using a Hall probe. Bulk magnetite was inserted afterwards into a liquidnitrogen-cooled Oxford Instruments Microstat with a fused silica window. Due to lack of a two-stage thermal shielding to allow for normal incident optical access and narrow design of the optical cryostat to fit into the magnet, substantial differences in sample and control sensor temperature occurred. To account for that the resistance of the sample was measured simultaneously and the Verwey transitions in the sample were used to linearly correct the differences between sensor and sample temperature. For comparison, electrical measurements of the Verwey transition on the same bulk sample in a Scientific Products Ltd. SRDK101E-HC-4E helium cryostat with a closed cycle refrigerator (CCR), resulting in a $T_{v}$ of $122 \pm 1 \mathrm{~K}$ for the crystal. The sample resistance was measured with a Keithley 2400 source meter.

\section{RAS-MOKE MEASUREMENTS}

The reflection of light from a surface produces changes in its state of polarization. Generally, if the incident beam is linearly polarized, the outgoing one is elliptically polarized and its polarization axis is rotated. Three different causes can produce such changes: anisotropy, chirality, and magnetism. The different contributions can be distinguished by their symmetry differences. In particular, the RAS response changes sign upon a sample rotation of $90^{\circ}$, MOKE changes sign upon magnetic field reversal, while a chiral response is unaffected by either change. By performing measurements at two azimuthal sample orientations at both magnetic field polarities, the components can be extracted. In the absence of a chiral response, as is the case for $\mathrm{Fe}_{3} \mathrm{O}_{4}(110)$, only two measurements, either with field reversal or sample rotation, are required to extract polar MOKE and RAS [36].

The RAS signal is defined as the difference in the complex Fresnel reflectivity along two in-plane orthogonal directions at near normal incidence:

$$
\frac{\Delta \tilde{r}}{\tilde{r}}=2 \frac{\tilde{r}_{x}-\tilde{r}_{y}}{\tilde{r}_{x}+\tilde{r}_{y}} .
$$

The RAS signal is then different from zero whenever the sample is in-plane anisotropic. In our case, indices $x$ and $y$ in Eq. (1) represent the direction along $\langle 001\rangle$ and $\langle 1 \overline{1} 0\rangle$, respectively.

MOKE also leads to a rotation in the polarization, however, in contrast to the structural optical anisotropy measured by RAS, the MOKE signal does depend on the magnetization of the sample. The two contributions can be effectively distinguished in linear optics. As the amplitudes are generally very small (below 5\%), the polarization rotations caused by RAS and MOKE are linearly superimposed.

If no external magnetic field is available, the RAS and any remanent polar MOKE can be separated by measuring two raw spectra, one where the incoming polarization is oriented $45^{\circ}$ with respect to the main sample axis $\left(M_{45}\right)$, and one after a $90^{\circ}$ rotation of the sample $\left(M_{-45}\right)$. A $90^{\circ}$ rotation of the sample around the surface normal produces a change in the sign of the RAS signal as geometrically the $x$ and $y$ axes are interchanged. The MOKE, however, remains unchanged, as it originates from a rotation of the incoming polarization around the out-of-plane magnetic field component, which is invariant to rotation around the surface normal. From two raw measurements $M_{45}, M_{-45}$, we can then easily extract the RAS and MOKE signal [36,37]:

$$
\begin{aligned}
\text { RAS } & =\left(M_{45}-M_{-45}\right) / 2, \\
\text { MOKE } & =\left(M_{45}+M_{-45}\right) / 2 .
\end{aligned}
$$

However, the MOKE signal extracted this way is only the polar component, measured in remanence. If MOKE hysteresis loops are to be measured, or the sample shows no out-of-plane remanent magnetization required for a polar MOKE signal, an external magnetic field is required to measure polar MOKE. To facilitate this, the electromagnet needs to have a gap or 
bore to allow for the optical measurements. In a setup with a magnet, the RAS and MOKE signatures can be separated even more easily and without any sample reorientation. A change in the direction of magnetization produces only a change in the sign of the Kerr signal. The RAS and MOKE signal can therefore be separated by two raw measurements with opposite magnetic field sign $\left(M_{+}, M_{-}\right)$:

$$
\begin{aligned}
\operatorname{RAS} & =\left(M_{+}+M_{-}\right) / 2, \\
\mathrm{MOKE} & =\left(M_{+}-M_{-}\right) / 2 .
\end{aligned}
$$

\section{RESULTS AND DISCUSSION}

\section{A. RAS of the bulk magnetite (110) surface}

We have initially measured the dependency of the RAS response on the azimuthal direction for the bulk sample. As expected, the largest RAS signal was measured when the directions $x$ and $y$ in Eq. (1) coincided with the two main orthogonal symmetry axes of the (110) surface: $\langle 001\rangle$ and $\langle 1 \overline{1} 0\rangle$. The real part of the RAS response of bulk magnetite (110) is shown in Fig. 1.

The real part of the RAS has a sharp positive peak at $1.1 \mathrm{eV}$, a negative one at $1.7 \mathrm{eV}$, and a large broad structure at $\sim 3 \mathrm{eV}$ : positive denotes a higher reflectivity for light polarized along the $\langle 100\rangle$ direction $(x)$.

No bulk anisotropy is expected from cubic crystals. This is also the case for (110) oriented crystals, as atomic layers with (110) alignment are rotated by $90^{\circ}$ with respect to each other. Thus, although the individual (110) layers have an anisotropy, the overall bulk crystal does not. However, (110) surfaces frequently do show optical anisotropies due to surface reconstructions or internal electrical fields caused by Fermi level pinning at the surface or by surface oxidization [25,40,41]. In the case of $\mathrm{Fe}_{3} \mathrm{O}_{4}(110)$, measured in air from a mechanically polished surface, we do not expect surface reconstructions to be responsible for the optical anisotropy. Instead, anisotropic strain or internal electrical fields are possible underlying causes. In this case, a close relationship between the RAS line shape with the dielectric function of the material is expected. In a simple three-layer model, the RAS response of an anisotropic dielectric layer with thickness $d$ on an isotropic bulk $\left(\tilde{\varepsilon}_{\mathrm{b}}\right)$ is related to the differences in the dielectric function $\left(\tilde{\varepsilon}_{x x}-\tilde{\varepsilon}_{y y}\right)$ of the anisotropic region as follows [25]:

$$
\frac{\Delta r}{r}=\frac{4 \pi i d}{\lambda} \frac{\tilde{\varepsilon}_{x x}-\tilde{\varepsilon}_{y y}}{\tilde{\varepsilon}_{\mathrm{b}}-1} .
$$

The real part of the RAS is hence linked to the imaginary part of $\tilde{\varepsilon}$, and vice versa. The RAS line shape resembles the dielectric function $(\tilde{\varepsilon})$, or its derivatives in energy [40], due to the occurrence in the term $\left(\tilde{\varepsilon}_{x x}-\tilde{\varepsilon}_{y y}\right)$. If $\tilde{\varepsilon}_{x x}$ and $\tilde{\varepsilon}_{y y}$ only differ in amplitude, the RAS will resemble $\tilde{\varepsilon}$. If $\tilde{\varepsilon}_{x x}$ and $\tilde{\varepsilon}_{y y}$ have similar amplitude, but differ slightly in energy due to, e.g., uniaxial strain, the RAS will resemble $d \tilde{\varepsilon} / d E$. This general observation applies to a response dominated by modified bulk states. Once surface reconstructions are involved, such an approach is generally not valid. Figure 1 compares the measured RAS with the first derivative of the dielectric function as measured by Schlegel et al. [7] and Fontijn et al. [10]. The correspondence between the RAS spectra and prominent features (maxima or minima) of the dielectric function of the material is evident.

Generally speaking, in any cubic structure, any termination anisotropy is canceled by $90^{\circ}$ rotation of adjacent planes. So, no RAS signal would be a priori expected. Anisotropies, as the one measured for $\mathrm{Fe}_{3} \mathrm{O}_{4}$ (110), are hence generated on the surface of the sample, either by anisotropies in the crystal field of the relaxed surface layer or electric field or strain gradients caused by the surface relaxation and oxidization.

In the presence of an electric field gradient a net anisotropy can occur, as the two rotated planes see a different electrical
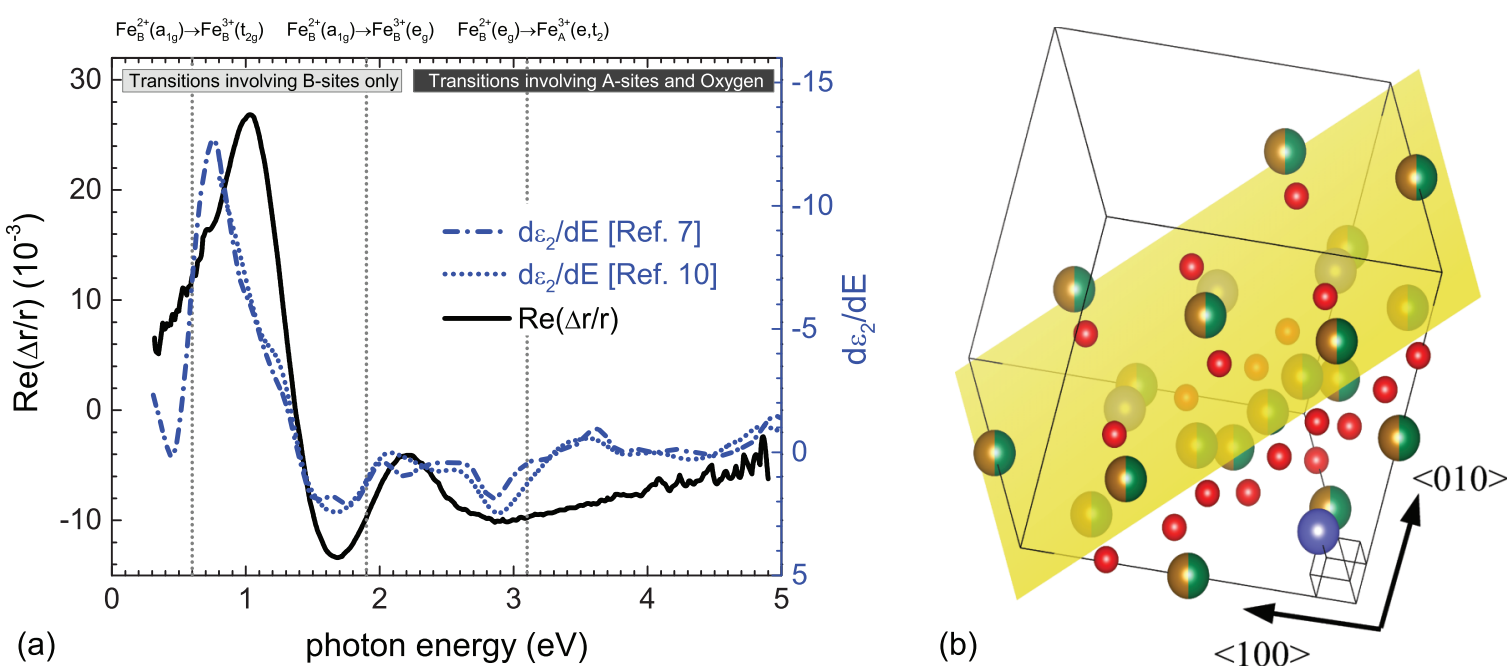

FIG. 1. (Color online) (a) Real part of the RAS spectra of single-crystal bulk magnetite $\mathrm{Fe}_{3} \mathrm{O}_{4}(110)$. For comparison, the first derivative of the imaginary part of the dielectric function of $\mathrm{Fe}_{3} \mathrm{O}_{4}$ is shown as reference (data taken from Schlegel et al. [7] and Fontijn et al. [10]). Vertical dotted lines denote energy positions of optical transitions involving states assigned to particular sites [10,39]. (b) Sketch of the unrelaxed B-layer terminated (110) surface of the $\mathrm{Fe}_{3} \mathrm{O}_{4}$ unit cell. The $\mathrm{O}$ atoms (red), the A-site Fe ions (violet), and the B-site Fe ions (green and brown) are depicted. The (110) surface is depicted in yellow. As the surface is anisotropic, the relaxed surface ions are subject to a different crystalline field compared to the bulk, resulting in the strong RAS signal measured. 
field, breaking the symmetry. A classic example of this effect is the dependency of certain RAS structures in GaAs on the surface termination and bulk doping levels [42]. However, as $\mathrm{Fe}_{3} \mathrm{O}_{4}$ is a semimetal, such field gradients could only exist if the presence of the surface leads to a reduction of the electron density at the Fermi level by lifting the degeneracy of the valence band maximum with the conduction band minimum. On the other hand, if surface atoms are relaxed, the crystal field for the top atoms is modified. Due to the anisotropy of the (110) surface, the relaxation induces different changes in the transition energies and probabilities along the $\langle 001\rangle$ and $\langle 1 \overline{1} 0\rangle$ directions, resulting in an anisotropic optical response. The surface anisotropy can also be induced by surface oxidization. For example, the first RAS measurements have been performed on a $\mathrm{Si}(110)$ surface, where the presence of the native oxide introduces strain in the underlying near surface bulk [43]. The observed RAS could be finally caused by strain or electric field gradients introduced by a thin native top oxide layer. As samples are measured in ambient air, $\mathrm{Fe}_{3} \mathrm{O}_{4}$ can form thin maghemite overlayers [44,45].

Due to its surface sensitivity, RAS signals can provide insight on the termination and structural properties of the $\mathrm{Fe}_{3} \mathrm{O}_{4}(110)$ surface. In the absence of $a b$ initio RAS calculations of the $\mathrm{Fe}_{3} \mathrm{O}_{4}(110)$ surface, a comparison to optical transitions in the bulk material can be used to discuss some potential origins of features in the reflectance anisotropy spectra. The observed structures in $\tilde{\varepsilon}$ have been assigned by Fontijn et al. to intervalence and intersublattice transitions by careful studies involving $\mathrm{Mg}^{2+}$ and $\mathrm{Al}^{3+}$ substituted $\mathrm{Fe}_{3} \mathrm{O}_{4}$. This was later confirmed by Antonov et al. in a calculation using the local spin-density approximation with onsite Coulomb interaction $(\mathrm{LSDA}+U)$ [39]. The observed optical transitions below $2.5 \mathrm{eV}$ are $d-d$ transitions made possible by significant mixing of iron $p$ and $f$ states in the initial and final states of the strongly correlated system. Most relevant for the RAS are transitions already seen in the reflectance data; a first structure, found experimentally at $0.6 \mathrm{eV}$, was assigned to a transition of spin-up states $\mathrm{Fe}_{\mathrm{B}}^{2+}\left(a_{1 g}\right)$ $\rightarrow \mathrm{Fe}_{\mathrm{B}}^{3+}\left(t_{2 g}\right)$. A second structure, experimentally at $1.9 \mathrm{eV}$, was assigned to spin-up transitions between $\mathrm{Fe}_{\mathrm{B}}^{2+}\left(a_{1 g}\right) \rightarrow \mathrm{Fe}_{\mathrm{B}}^{3+}\left(e_{g}\right)$. The first calculated transition involving $\mathrm{A}$ sites was within spin-down states $\mathrm{Fe}_{\mathrm{B}}^{2+}\left(e_{g}\right) \rightarrow \mathrm{Fe}_{\mathrm{A}}^{3+}\left(e, t_{2}\right)$ at an energy of $2.5 \mathrm{eV}$. In measurements, such a third absorption peak was observed only around $3.1 \mathrm{eV}[10,11]$. While $a b$ initio calculations can provide valuable insight into the origin of structures in $\tilde{\varepsilon}$, the transition energies are not correctly reproduced yet. Figure 1 shows that the most dominant RAS feature is found in the energy range of transitions involving the $\mathrm{B}$-site $\mathrm{Fe}^{2+}$ and $\mathrm{Fe}^{3+}$ ions and significantly below the lowest-energy transition associated to ions on the A site, suggesting a B-site sensitivity of the RAS spectra.

Recently, first-principles calculations of different magnetite surfaces were carried out and the relaxation of the top atoms was predicted [47]. The favorable structures for both A- and B-site terminated (110) surfaces were obtained and their total energies were found to be comparable. The B-site sensitivity of the RAS suggests that the presence of the surface dominantly distorts the octahedral sites. This could indicate that the actual surface is terminated by these B sites, as in this case any

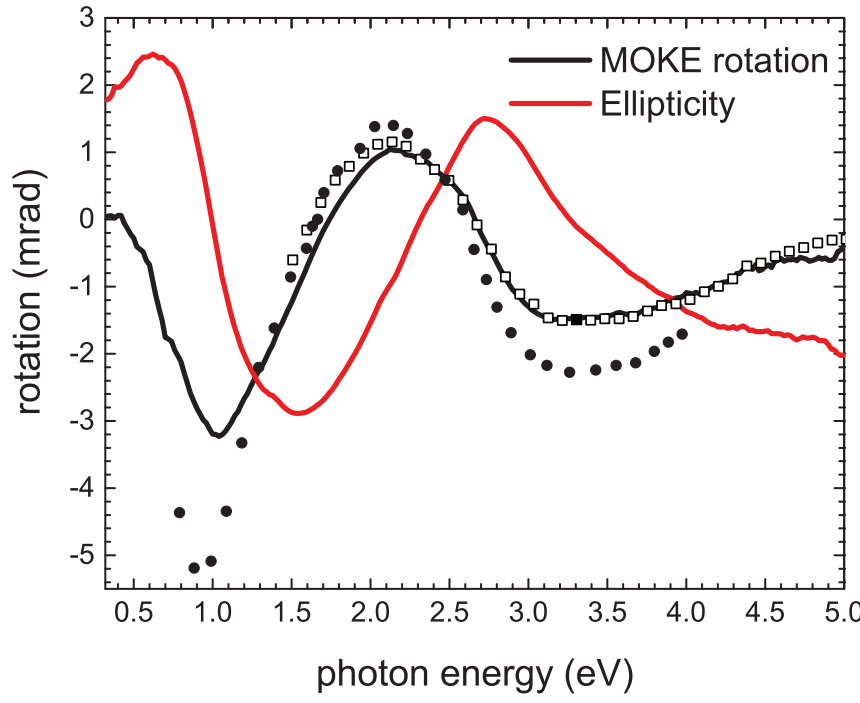

FIG. 2. (Color online) MOKE rotation and ellipticity of bulk $\mathrm{Fe}_{3} \mathrm{O}_{4}(110)$. For comparison, published data from Kim et al. [46] (open squares) and Fontijn et al. [10] (full circles) are included.

surface-induced crystal field distortion should be maximized. Further first-principles calculations of the optical transition probabilities of the surface would be required to understand the anisotropic optical response of the $\mathrm{Fe}_{3} \mathrm{O}_{4}(110)$ surface more fully and possibly differentiate between A- or B-site termination.

As outlined, the experimental setup detects both the optical anisotropy and polar MOKE signal. To confirm the correct extraction of the RAS and MOKE signals, Fig. 2 compares the MOKE response of a bulk sample to previously published data [46]. Our data in the infrared region are equally consistent with earlier reports, except for differences in amplitude consistent with those found between natural and synthetic $\mathrm{Fe}_{3} \mathrm{O}_{4}$ crystals $[33,35]$.

\section{B. RAS measurements across the Verwey transition}

$\mathrm{Fe}_{3} \mathrm{O}_{4}$ undergoes a metal-insulator transition at around $T_{v} \sim 120 \mathrm{~K}$. This so-called Verwey transition has been attributed to charge ordering of the structure, which causes a reduction of the symmetry of the crystal from the roomtemperature cubic phase. Below $T_{v}$, the Fe ions in the B sites rearrange along the $\langle 1 \overline{1} 0\rangle$ and the $\langle 110\rangle$ directions producing a distortion of the crystal [19], which results in charge accumulation at certain sites in the $\mathrm{Fe}_{3} \mathrm{O}_{4}$ lattice. The details of how these atomic displacements influence the electronic structure of the material are nevertheless still actively discussed and many models have been considered [17-20,48].

RAS spectra of $\mathrm{Fe}_{3} \mathrm{O}_{4}(110)$ show structures in the energetic range of electronic transitions involving the $\mathrm{B}$ sites and RAS spectra from this spectral region were recorded across the Verwey transition. Figure 3(a) shows that the intensity of the RAS peaks increases as temperature decreases, while the higher-energy region appears almost unaffected. The steady increase in the intensity of the RAS spectrum with decreasing temperature is attributed to the decrease in vibrational 

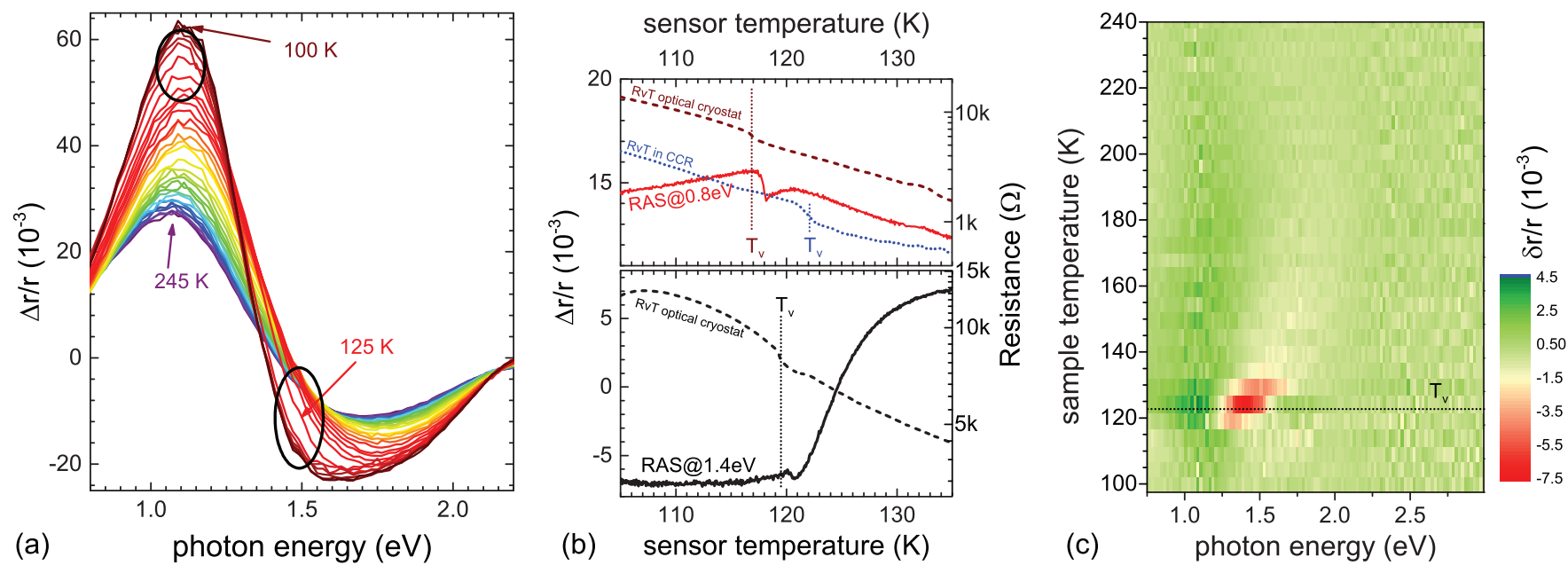

FIG. 3. (Color online) (a) Real part of the RAS spectrum of bulk magnetite measured between 240 and $100 \mathrm{~K}$ in 5-K steps. The lower the temperature, the higher the RAS amplitude. The circles highlight more sudden changes in specific spectral ranges upon the Verwey transition. (b) Shows RAS transients taken at 1.4 and $0.8 \mathrm{eV}$ during a sample warmup with $2 \mathrm{~K} / \mathrm{min}$ and simultaneous resistance measurement as reference. The true sample temperature was estimated by offsetting the sensor temperature by the measured different in $T_{v}$ of each transient with the reference measurement in the shielded electrical cryostat (CCR). (b) Difference between RAS spectra measured at two adjacent temperature steps. A significantly larger change in the RAS signal is measured just above the Verwey temperature. Difference spectra have been color coded for better visibility of the effect.

broadening of the optical transitions resulting in a sharpening and blue-shift in energy $[11,49]$.

In contrast, there is an abrupt increase in the peak intensity of the strongest anisotropic feature at $\sim 1 \mathrm{eV}$ and a noticeable shape change around $1.4 \mathrm{eV}$ below $120 \mathrm{~K}$ [see circles in Fig. 3(a)]. Figure 3(c) highlights this abrupt change by comparing differential RAS spectra from adjacent temperature steps with 5-K temperature resolution. The RAS transients [Fig. 3(b)] taken at 0.8 and $1.4 \mathrm{eV}$ reveal that the biggest change in the RAS occurs just above the occurrence of the typical resistance change associated to $T_{v}$. At $0.8 \mathrm{eV}$, the largest change is just $1 \mathrm{~K}$ above that, while for the transient taken at $1.4 \mathrm{eV}$, the largest change in RAS is observed $\sim 5 \mathrm{~K}$ above $T_{v}$. This finding supports the recent suggestion from Raman spectra that the Verwey transition is a two-step process with a crystallographic and charge reorder not happening simultaneously [20].

There are previous reports of a sudden reduction in the reflectivity far infrared $\left(\sim 480 \mathrm{~cm}^{-1}\right)$ [11,50]. In this spectral range, the free-electron absorption and vibrational modes of the magnetite crystal dominate the response, but the value is well below the energy range analyzed in this study. Nevertheless, as optical transitions are related to each other through electron sum rules, simultaneous changes in other spectral ranges can be a priori expected. Similar effects have been previously seen in RAS of photobleaching in superconductors [32] or in metal-insulator transitions of surface reconstructions [51-53]. In a similar way, the expected opening of the band gap and consequently reduction in reflection in the infrared region should lead to an increased reflection elsewhere. Indeed, such behavior was observed in wide spectral range measurements [11]. However, in the spectral range associated to transitions involving the B-site $\mathrm{Fe}^{3+}$ and $\mathrm{Fe}^{2+}$ ions, the temperature-dependent reflectance measurements are dominated by the sharpening of optical transitions and reduction in free-electron absorption $[11,50]$.

In comparison, the RAS spectra reported here are significantly more sensitive to changes in the electronic structure and changes in the RAS spectra are quite large with respect to the total RAS signal. As shown in Fig. 4, the peak-to-peak amplitude changes from $68 \times 10^{-3}$ at $135 \mathrm{~K}$ to $80 \times 10^{-3}$ at $115 \mathrm{~K}$. It is intriguing to relate this change of $18 \%$ to the charge or orbital ordering involving the $\mathrm{B}$-site $\mathrm{Fe}^{3+}$ and

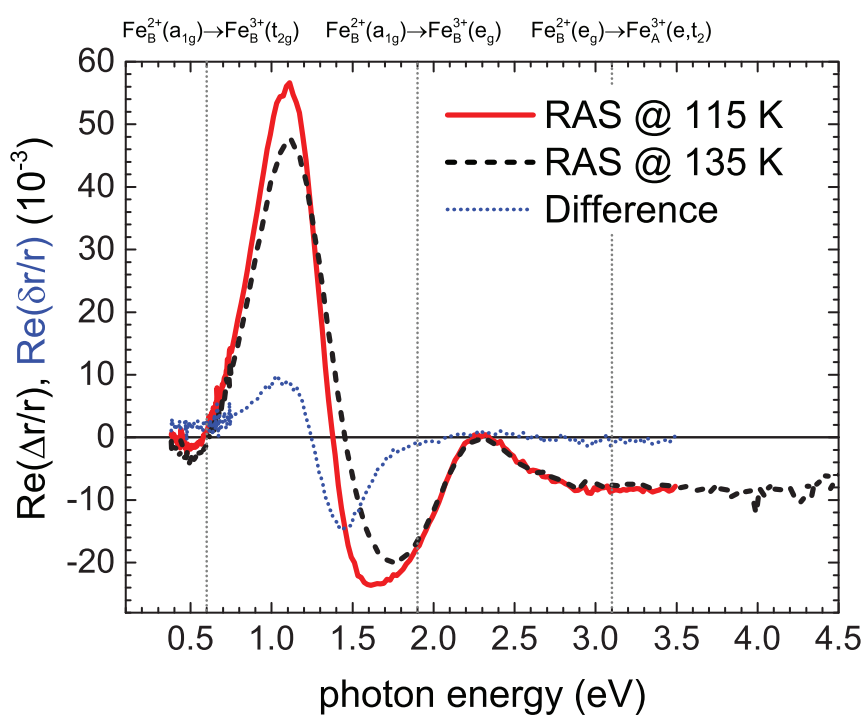

FIG. 4. (Color online) Full spectral range RAS spectra of a single-crystal bulk magnetite (110) at $135 \pm 5 \mathrm{~K}$ (red line) and $115 \pm$ $5 \mathrm{~K}$ (black, dashed line) and the difference between them (blue, dotted line). Upon the Verwey transition, the shape and amplitude of the RAS signature changes significantly. 
$\mathrm{Fe}^{2+}$ ions. As discussed earlier, the most prominent change in the RAS occurs in the spectral region associated with optical transitions involving these sites. It is possible that, below $125 \mathrm{~K}$, the change in peak-to-peak RAS amplitude is associated with a corresponding charge order at the $\mathrm{Fe}^{2+}$ and $\mathrm{Fe}^{3+}$ sites and associated changes in the density of states of the ground and final states. A $b$ initio calculations of the optical anisotropy are needed for the RT and LT phases to confirm this assignment. Such calculations could distinguish between the various competing models of this charge ordering, as they would need to be able to reproduce our experimental findings. For other metal-insulator transitions, such as the Peierls transition of In-Si(111)- $(4 \times 1)$, structural changes have been identified by a direct comparison of RAS data with $a b$ initio calculations $[51,53,54]$. Due to the self-normalizing nature of an RAS spectra, we are not only more sensitive to changes in the electronic configuration, compared to absolute reflectance measurements, it can also be easier to quantitatively compare DFT calculations of RAS spectra to measurements, as some inherent limitations in the calculations can be canceled in calculating the difference in reflectance of the two orthogonal directions and the normalization by the average reflectance. While computationally more expensive due to the significantly larger unit cells required for surface calculations, the quantitative agreement in RAS calculations with experiments can be quite good, making the technique ideal to test different surface or electronic ordering models $[41,51,55]$.

\section{Thin-film optical response}

So far, we have discussed the anisotropic optical response of a mechanically polished bulk sample. In this section, we will apply RAS as a fingerprint tool to monitor the growth of magnetite thin films on nearly lattice-matched $\mathrm{MgO}(110)$ substrates. Figure 5 shows the comparison between bulk magnetite and the thickest film prepared $(75 \mathrm{~nm})$. While there are similarities, the overall shape and intensity of the RAS response of epitaxial $\mathrm{Fe}_{3} \mathrm{O}_{4}(110)$ films on $\mathrm{MgO}$ is different from the bulk. As the penetration depth of light for magnetite varies from just $30 \mathrm{~nm}$ in the UV to a maximum of $160 \mathrm{~nm}$ around $1.4 \mathrm{eV}$ the measured RAS response of a thin-film sample will differ from a bulk sample, even if the surface termination and hence the surface dielectric anisotropy (SDA) $\Delta \varepsilon d$ is identical. For a thin-film sample, however, the RAS can not be simply modeled with equation (4), as the latter is only valid in a three-layer system. Full optical simulations using a transfer-matrix approach for anisotropic media are required [24,56-59]. In Fig. 5, such a model is shown.

First, the SDA of the bulk sample was fitted using Eq. (4) in order to reproduce the bulk anisotropy [see Fig. 5(a)]. The thickness of the anisotropic surface region $d$ was assumed to be $1.5 \mathrm{~nm}$. As only $\Delta \varepsilon d$ can be derived from RAS, the thickness value $d$ is an input parameter in the simulation. The choice of $1.5 \mathrm{~nm}$, however, is not arbitrary: It was the smallest value of $d$, where the absolute values of the surface $\tilde{\varepsilon}_{y y}$ required to fit our RAS data did not vary more than $5 \%$ from $\tilde{\varepsilon}_{x x}$ fixed at published bulk values. Fundamentally, for thickness values well below the wavelength it is impossible to decouple $\varepsilon d$ or $\Delta \varepsilon d$ with any optical technique [60].
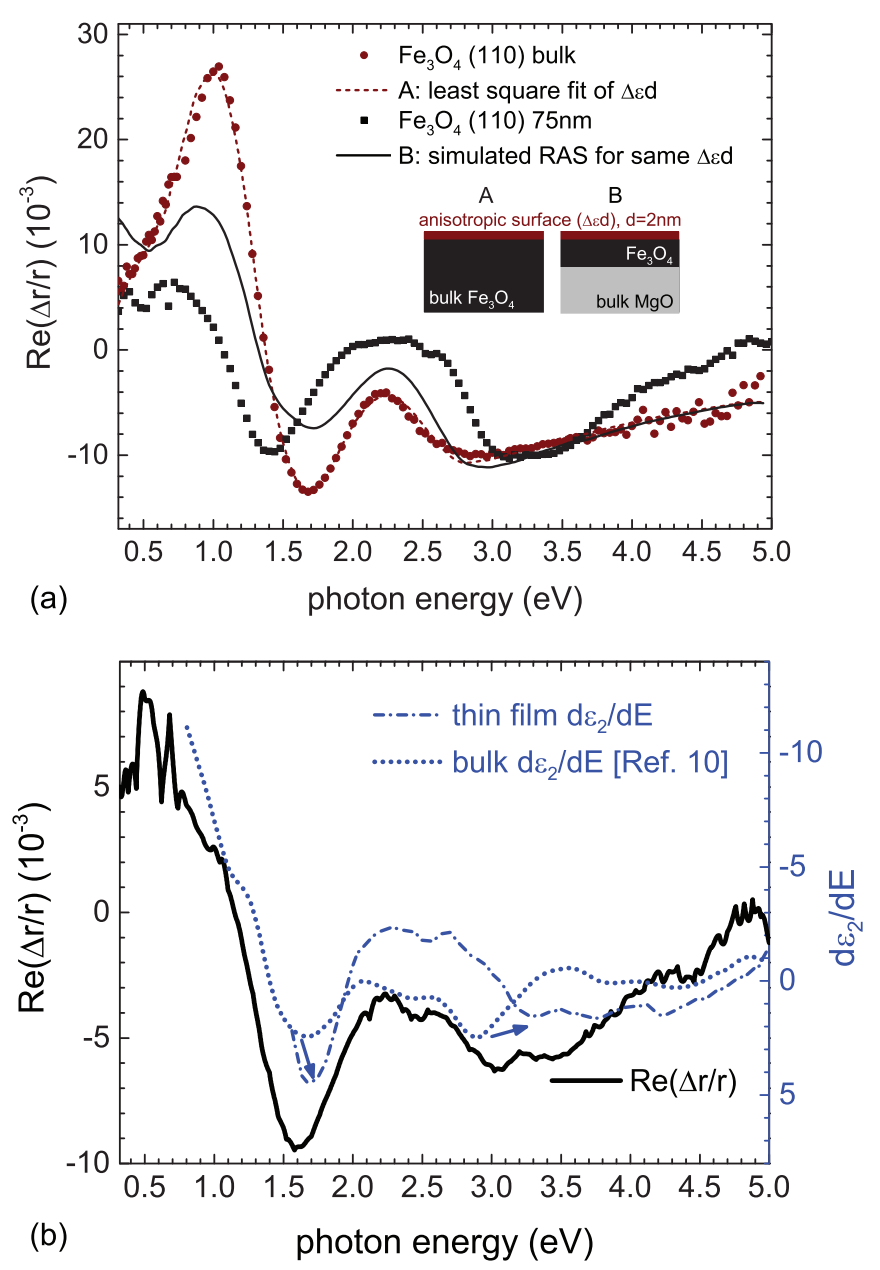

FIG. 5. (Color online) (a) Room-temperature RAS spectra of a single-crystal bulk magnetite (110) sample (circles) and of a 75-nm film grown on $\mathrm{MgO}$ (110) by MBE (squares). The dashed line is an optical model of the bulk sample using the bulk dielectric function of $\mathrm{Fe}_{3} \mathrm{O}_{4}$ and a least-square fit of a modeled surface dielectric anisotropy $(\mathrm{SDA}=\Delta \varepsilon d)$. The solid line uses the same SDA to model the thinfilm sample using no additional parameters. (b) Comparison of the RAS of a 31-nm-thick thin-film sample with the first derivative of the bulk $\mathrm{Fe}_{3} \mathrm{O}_{4}$ dielectric function and the thin-film dielectric function as measured on the same film.

In a second step, the same SDA was used to simulate

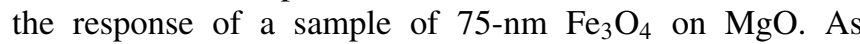
seen in Fig. 5, the reduced amplitude of the RAS is already reproduced. The main changes in amplitude are hence related to changes in the reflectance of the stacked sample. The additional differences could arise from the different dielectric function of the thin-film sample when compared to the bulk. We therefore also measured RAS of a 31-nm-thick film and extracted the dielectric function by ellipsometry using the known thickness of the sample measured by XRR. As seen in Fig. 5(b), the RAS response of the thin film, within the limited spectral range of the ellipsometer, resembles the first derivative of the thin-film $\mathrm{Fe}_{3} \mathrm{O}_{4}$ dielectric function, similar to the behavior of the bulk sample (see Fig. 1). However, prominent structures are blue-shifted (marked with arrows) compared to the bulk dielectric function. The changes in RAS 


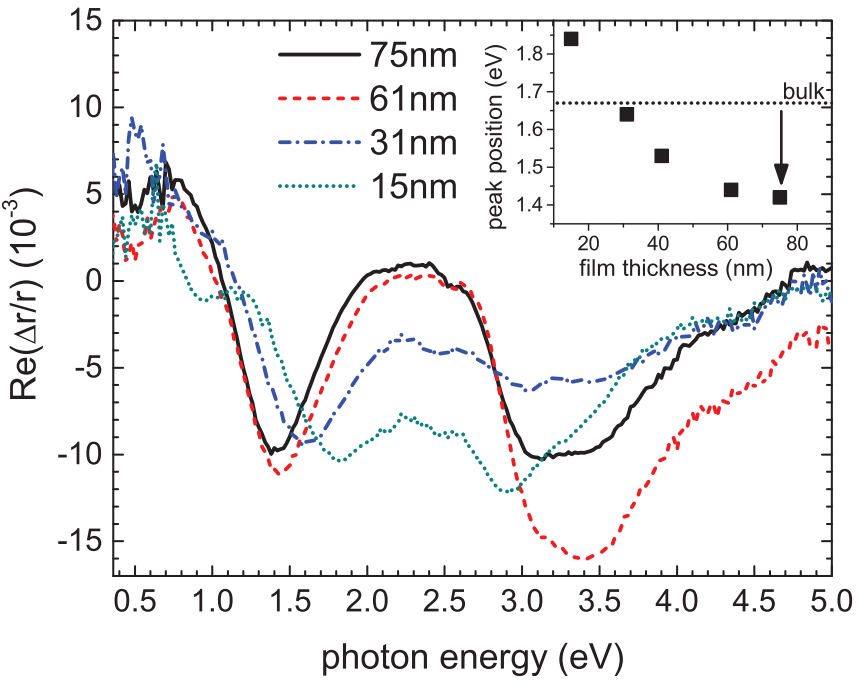

FIG. 6. (Color online) RAS spectra of (110) films measured for different film thickness. The inset shows the position of the first negative $\langle 1 \overline{1} 0\rangle$ peak as a function of the film thickness compared to the position in the bulk sample (dotted line).

are hence not necessarily caused by differences in the surface termination, but rather by changes in the thin-film dielectric function itself.

A systematic study of the RAS spectra for different film thickness of $\mathrm{Fe}_{3} \mathrm{O}_{4}(110)$ has been finally performed (see Fig. 6). Films having different thickness present different RAS signature. Even without a detailed knowledge on the cause of the anisotropic signal, the RAS signature can be used to monitor the growth of magnetite (110) films in situ. In particular, there is a clear correspondence of the RAS minima position with thickness (see inset Fig. 6) which can be used as a fingerprint to follow the growth evolution. RAS measurement can be performed in a short time, it is a nondestructive method, and produced a strong signal even for films as low as $15 \mathrm{~nm}$, rendering it appealing for practical applications. The technique has been already used for the growth of different heteroepitaxial structures [24], and our data are a proof of principle that RAS can also be applied to monitor oxide growth.

More detailed interpretation of the thin-film RAS response is not straightforward. Furthermore, compared to the bulk counterpart, the anisotropy of the thin films could also arise from bulk uniaxial strain in the epitaxial system. XRD was then used to measure the epitaxial nature of the layers and also the strain relaxation. The latter indicates how much the epitaxial strain caused by the lattice mismatch of $\mathrm{Fe}_{3} \mathrm{O}_{4}$ with respect to the $\mathrm{MgO}(110)$ substrate is relieved by dislocations or other defects. A value of $0 \%$ means a fully strained film, while $100 \%$ indicates a fully relaxed film, showing the same lattice parameter as bulk $\mathrm{Fe}_{3} \mathrm{O}_{4}$. Only the out-of-plane $d$ spacing ([110]) and one in-plane constant $a_{\|}$(along [001]) could be determined by XRD. For better comparison, we converted the $d$ spacing of the [110] planes into an effective lattice parameter $a_{\perp}=4 \sqrt{2} d_{[440]}$. The results are presented in Table I and it can be seen that very little strain relaxation was observed, even for the thicker layers.
TABLE I. Dependency of $\mathrm{Fe}_{3} \mathrm{O}_{4}$ lattice constants and position of the first RAS peak for different film thickness. The effective out-ofplane lattice constant $a_{\perp}$ was determined by symmetric $2 \theta$ scans of the $\mathrm{Fe}_{3} \mathrm{O}_{4}$ [440] reflex. The in-plane lattice constant $a_{\|}$was determined by reciprocal mapping of the [222] reflex. $s_{R}$ denotes the strain relaxation of the film in $\%$. The corresponding database values of unstrained bulk material are given in the first two rows. Numbers in brackets denote the error in the last figure.

\begin{tabular}{lcccc}
\hline \hline $\begin{array}{l}\text { Thickness } \\
(\mathrm{nm})\end{array}$ & $\begin{array}{c}\text { RAS peak } \\
(\mathrm{eV})\end{array}$ & $\begin{array}{c}a_{\|} \\
(\AA)\end{array}$ & $\begin{array}{c}a_{\perp} \\
(\AA)\end{array}$ & $\begin{array}{c}s_{R} \\
(\%)\end{array}$ \\
\hline $\mathrm{MgO}$ & & 4.2112 & $=a_{\|}$ & \\
$\mathrm{Fe}_{3} \mathrm{O}_{4}$ & & 8.3778 & $=a_{\|}$ & 100 \\
75 & 1.38 & $8.422(3)$ & $8.165(1)$ & $2.9(1)$ \\
65 & 1.44 & $8.421(5)$ & $8.162(3)$ & $2.3(2)$ \\
31 & 1.57 & $8.422(8)$ & $8.163(5)$ & $1.4(5)$ \\
15 & 1.78 & $\mathrm{a}$ & $8.16(3)$ & $\mathrm{a}$ \\
\hline
\end{tabular}

a Note that for the thinnest 15-nm film, the [222] reflex was too broad and weak to determine the in-plane lattice constant and strain relaxation.

The effect of strain on the RAS could be considered by a three-layer system and using a modified, now anisotropic, bulk dielectric function and the $\mathrm{Fe}_{3} \mathrm{O}_{4}$ film thickness as measured by XRR. In this case, the anisotropy is generated though the whole magnetite layer. Within this model, the RAS of the thickest (75-nm) sample can be described using the measured film thickness, the $\mathrm{Fe}_{3} \mathrm{O}_{4}$ bulk dielectric function for $\varepsilon_{x x}$, and a least-square fit of a modified dielectric function for $\varepsilon_{y y}$ where the energetic positions, amplitudes, and broadening of each oscillator is used as fitting parameter. Figure 7(a) shows the expected RAS changes for thinner layers using the same $\varepsilon_{x x}$ and $\varepsilon_{y y}$, describing a system where the strain is similar for each thickness as supported by the XRD strain measurements listed in Table I. This model partly explains the observed blue-shift of the RAS minimum for thinner layers. The magnitude of the blue-shift, the observed amplitude reduction, and the pronounced changes in the UV are not reproduced. In particular, the RAS between $2-2.5 \mathrm{eV}$ would always be expected to be 0 as it is for the 75-nm sample. This is clearly not the case for thinner films.

The changes in the energetic region above $2 \mathrm{eV}$ are qualitatively reproduced by a second model, assuming that the anisotropy is generated only in the surface layer, having an SDA identical to that of the bulk sample. In this case, shown in Fig. 7(b), an increase in RAS amplitude would be expected for thinner layers, as the SDA is constant but the overall stack reflectivity decreases with the reduction in $\mathrm{Fe}_{3} \mathrm{O}_{4}$ thickness, particularly in the spectral range where the penetration depth is large. The model also shows that the expected changes due to the multilayer reflectance changes will not change the RAS minimum positions.

Hence, based on these simulations, we can infer that only a combination of the effects presented here could explain all the features observed. However, to fully model this, we not only have to describe the dielectric anisotropy $\Delta \varepsilon d$ but also the modified thin-film dielectric function $\varepsilon$ itself leaving too many unknown variables to derive information from the optical modeling. 
Model 1: Bulk anisotropy in epitaxial film

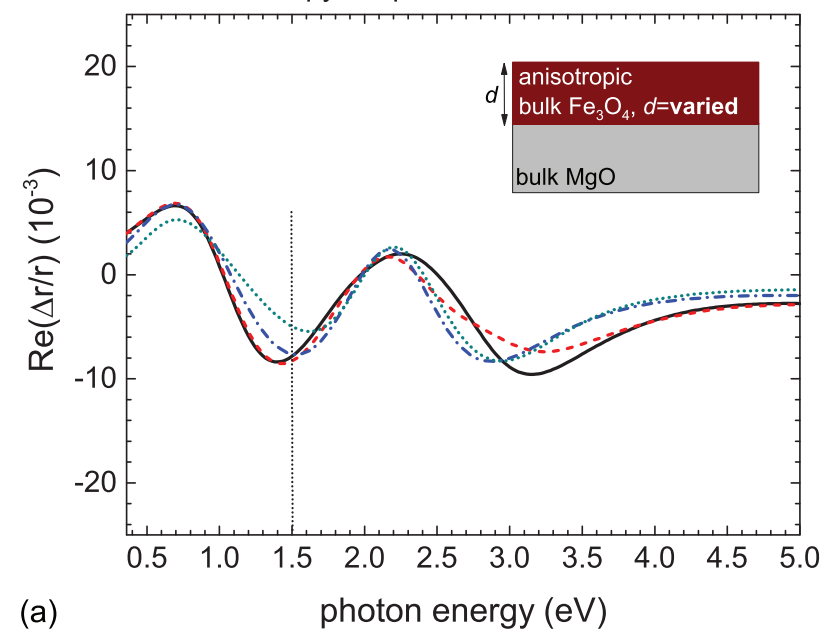

Model 2: Bulk like surface anisotropy and bulk dielectric function

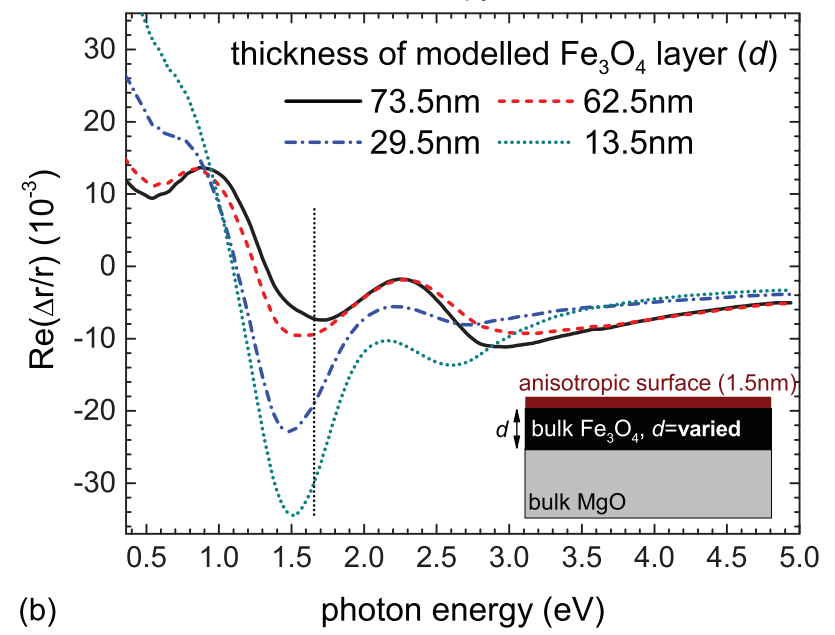

FIG. 7. (Color online) Simulated RAS spectra for various possible models describing the thin-film dielectric function. (a) Bulk anisotropic strain throughout the thin film, (b) a model assuming the same dielectric anisotropy in the thin-films samples as seen for the bulk sample. The dotted line indicates the RAS minimum position of the bulk $\mathrm{Fe}_{3} \mathrm{O}_{4}(110)$ surface as a reference.

A final point to discuss is the possible formation of an maghemite overlayer, and how it would affect the RAS. Previous ellipsometric measurements have estimated the formation of a top surface oxidization of up to $4 \mathrm{~nm}$ for $\mathrm{Fe}_{3} \mathrm{O}_{4}(001)$ thin films grown on various lattice-matched substrates [45]. The formation of such a surface maghemite [44] layer can not only be the cause of a RAS response on a (110) surface, but can also alter its shape due to the change in overall reflectivity in such a multilayered system. As samples in Fig. 6 have been measured ex situ, after different air exposure times it is possible that some of the observed changes are caused by varying surface maghemite layer thickness. However, as epitaxial maghemite only shows distinct peaks in its absorption spectra above $2 \mathrm{eV}$ [61], the discussed shift in RAS peak position (1.4 to $1.8 \mathrm{eV}$ ) can not be influenced by this. The RAS amplitude, however, could change, assuming a thicker oxide overlayer has a less ordered and therefore less anisotropic oxide/ $/ \mathrm{Fe}_{3} \mathrm{O}_{4}$ interface. Previous studies on 40- and 115-nm thick $\mathrm{Fe}_{3} \mathrm{O}_{4}$ on $\mathrm{MgAl}_{2} \mathrm{O}_{4}$, however, have not seen a significant difference in oxide overlayer thickness [45]. While no systematic studies on $\mathrm{Fe}_{3} \mathrm{O}_{4}$ grown on $\mathrm{MgO}(110)$ exist, preliminary results [62] have demonstrated that ultrathin films of 4-nm $\mathrm{Fe}_{3} \mathrm{O}_{4}$ thickness grown on $\mathrm{MgO}(001)$ show the presence of a surface maghemite layer already after one day of exposure, however, are not fully oxidized even after one year of exposure to ambient atmosphere, limiting the possible maghemite layer thickness to below 2-3 $\mathrm{nm}$. More extensive studies involving a comparison of in situ and ex situ measurements, as well as long-term time-dependent measurements upon air exposure would be required to clarify this point.

\section{v. CONCLUSIONS}

Optical anisotropies of $\mathrm{Fe}_{3} \mathrm{O}_{4}(110)$ from bulk and thin-film samples have been measured using RAS. The anisotropic and magnetic contributions were successfully separated, with the validity of the approach being demonstrated by comparing the MOKE response with previous published data. The RAS spectra resemble the derivative of the dielectric function of the samples, indicating that small shifts in energy of the optical transitions, such as those associated with anisotropic strain, are responsible for the anisotropic response. The changes in the RAS response across the Verwey transition have been measured and could be useful in clarifying the electronic structure of the orbital ordering and charge ordering if $a b$ initio calculations of the RAS spectra of the room-temperature and low-temperature phases become available. Finally, the RAS response of $\mathrm{Fe}_{3} \mathrm{O}_{4}(110)$ thin films grown on a $\mathrm{MgO}(110)$ substrate has been measured. Significant changes in the spectral response occur as the film thickness is varied between 15 and $75 \mathrm{~nm}$. The results suggest that RAS can be used as a nondestructive simple optical method to monitor the film growth in situ. All samples have been measured in ambient conditions and the measured reflectance anisotropy originates either from a surface anisotropy of a B-site terminated (110) surface, or an inherent anisotropy introduced by the surface oxidization of the (110) surface.

\section{ACKNOWLEDGMENTS}

This work has been funded by Science Foundation of Ireland, Contracts No. 06/IN.1/I91, No. 12/IA/1264, and No. 11/RFP.1/PHY/3047, and conducted under the framework of the INSPIRE programme, funded by the Irish Government's Programme for Research in Third Level Institutions, Cycle 4, National Development Plan 2007-2013. O. Mauit acknowledges support of the Program Bolashak funded by the government of Kazakhstan.
[1] H. Zeng, J. Li, J. P. Liu, Z. L. Wang, and S. Sun, Nature (London) 420, 395 (2002).
[2] R. Hiergeist, W. Andrä, N. Buske, R. Hergt, I. Hilger, U. Richter, and W. Kaiser, J. Magn. Magn. Mater. 201, 420 (1999). 
[3] P. A. Miles, W. B. Westphal, and A. Von Hippel, Rev. Mod. Phys. 29, 279 (1957).

[4] S. Lee, A. Fursina, J. T. Mayo, C. T. Yavuz, V. L. Colvin, R. G. Sumesh Sofin, I. V. Shvets, and D. Natelson, Nat. Mater. 7, 130 (2008).

[5] C. H. Li, Phys. Rev. 40, 1002 (1932).

[6] L. McGuigan, R. C. Barklie, R. G. S. Sofin, S. K. Arora, and I. V. Shvets, Phys. Rev. B 77, 174424 (2008).

[7] A. Schlegel, S. F. Alvarado, and P. Wachter, J. Phys. C: Solid State Phys. 12, 1157 (1979).

[8] I. Balberg and J. I. Pankove, Phys. Rev. Lett. 27, 596 (1971).

[9] A. J. M. Kuipers and V. A. M. Brabers, Phys. Rev. Lett. 39, 488 (1977).

[10] W. F. J. Fontijn, P. J. van der Zaag, M. A. C. Devillers, V. A. M. Brabers, and R. Metselaar, Phys. Rev. B 56, 5432 (1997).

[11] S. K. Park, T. Ishikawa, and Y. Tokura, Phys. Rev. B 58, 3717 (1998).

[12] W. H. Bragg, Philos. Mag. 30, 305 (1915).

[13] M. E. Fleet, Acta Crystallogr., Sect. B: Struct. Crystallogr. Cryst. Chem. 37, 917 (1981).

[14] Y. Fujii, G. Shirane, and Y. Yamada, Phys. Rev. B 11, 2036 (1975).

[15] M. Iizumi and G. Shirane, Solid State Commun. 17, 433 (1975).

[16] E. Verwey and P. Haayman, Physica (Amsterdam) 8, 979 (1941).

[17] F. Walz, J. Phys. C: Solid State Phys. 14, R285 (2002).

[18] V. N. Antonov, L. V. Bekenov, and A. N. Yaresko, Adv. Condens. Matter Phys. 2011, 298928 (2011).

[19] M. S. Senn, J. P. Wright, and J. P. Attfield, Nature (London) 481, 173 (2012).

[20] M. B. Yazdi, K.-Y. Choi, D. Wulferding, P. Lemmens, and L. Alff, New J. Phys. 15, 103032 (2013).

[21] H.-C. Wu, M. Abid, B. S. Chun, R. Ramos, O. N. Mryasov, and I. V. Shvets, Nano Lett. 10, 1132 (2010).

[22] N. Uekawa and K. Kaneko, J. Phys. Chem. B 102, 8719 (1998).

[23] J. P. Hong, S. B. Lee, Y. W. Jung, J. H. Lee, K. S. Yoon, K. W. Kim, C. O. Kim, C. H. Lee, and M. H. Jung, Appl. Phys. Lett. 83, 1590 (2003).

[24] J.-T. Zettler, Prog. Cryst. Growth Charact. Mater. 35, 27 (1997).

[25] P. Weightman, D. S. Martin, R. J. Cole, and T. Farrell, Rep. Prog. Phys. 68, 1251 (2005).

[26] D. E. Aspnes and A. A. Studna, Phys. Rev. Lett. 54, 1956 (1985).

[27] P. Hofmann, K. C. Rose, V. Fernandez, A. M. Bradshaw, and W. Richter, Phys. Rev. Lett. 75, 2039 (1995).

[28] I. Kamiya, D. E. Aspnes, L. T. Florez, and J. P. Harbison, Phys. Rev. B 46, 15894 (1992).

[29] K. Fleischer, L. Carroll, C. Smith, and J. F. McGilp, J. Phys. C: Solid State Phys. 19, 266003 (2007).

[30] D. S. Martin and P. Weightman, Surf. Inter. Anal. 31, 915 (2001).

[31] U. Rossow, R. Goldhahn, D. Fuhrmann, and A. Hangleiter, Phys. Status Solidi B 242, 2617 (2005).

[32] S. Bahrs, A. Bruchhausen, A. Goni, G. Nieva, A. Fainstein, K. Fleischer, W. Richter, and C. Thomsen, J. Phys. Chem. Solids 67, 340 (2006).

[33] X. Zhang, J. Schoenes, and P. Wachter, Solid State Commun. 39, 189 (1981).

[34] H. Boppart, A. Schlegel, and P. Wachter, Philos. Mag. B 42, 431 (1980).

[35] I. Müller and U. Buchenau, Phys. B+C (Amsterdam) 80, 69 (1975).
[36] T. Herrmann, K. Lüdge, W. Richter, K. G. Georgarakis, P. Poulopoulos, R. Nünthel, J. Lindner, M. Wahl, and N. Esser, Phys. Rev. B 73, 134408 (2006).

[37] J. P. Cunniffe, D. E. McNally, M. Liberati, E. Arenholz, C. McGuinness, and J. F. McGilp, Phys. Status Solidi B 247, 2108 (2010).

[38] S. K. Arora, R. G. S. Sofin, I. V. Shvets, and M. Luysberg, J. Appl. Phys. 100, 073908 (2006).

[39] V. N. Antonov, B. N. Harmon, V. P. Antropov, A. Y. Perlov, and A. N. Yaresko, Phys. Rev. B 64, 134410 (2001).

[40] U. Rossow, L. Mantese, and D. Aspnes, Appl. Surf. Sci. 123-124, 237 (1998).

[41] W. Schmidt, F. Bechstedt, and J. Bernholc, J. Vac. Sci. Technol. B 18, 2215 (2000).

[42] Z. Sobiesierski and D. Westwood, Thin Solid Films 318, 140 (1998.)

[43] D. E. Aspnes, J. Vac. Sci. Technol. B 3, 1498 (1985).

[44] F. Bourgeois, P. Gergaud, H. Renevier, C. Leclere, and G. Feuillet, J. Appl. Phys. 113, 013510 (2013).

[45] W. F. J. Fontijn, R. M. Wolf, R. Metselaar, and P. van der Zaag, Thin Solid Films 292, 270 (1997).

[46] K. J. Kim, H. S. Lee, M. H. Lee, and S. H. Lee, J. Appl. Phys. 91, 9974 (2002).

[47] T. Yang, X.-D. Wen, J. Ren, Y.-W. Li, J.-G. Wang, and C.-F. Huo, J. Fuel Chem. Tech. 38, 121 (2010).

[48] I. Leonov, A. N. Yaresko, V. N. Antonov, M. A. Korotin, and V. I. Anisimov, Phys. Rev. Lett. 93, 146404 (2004).

[49] M. Cardona, Solid State Commun. 133, 3 (2005).

[50] J. Ebad-Allah, L. Baldassarre, M. Sing, R. Claessen, V. A. M. Brabers, and C. A. Kuntscher, J. Appl. Phys. 112, 073524 (2012).

[51] K. Fleischer, S. Chandola, N. Esser, W. Richter, J. McGilp, W. Schmidt, S. Wang, W. Lu, and J. Bernholc, Appl. Surf. Sci. 234, 302 (2004).

[52] K. Fleischer, S. Chandola, N. Esser, W. Richter, and J. F. McGilp, Phys. Rev. B 67, 235318 (2003).

[53] S. Chandola, K. Hinrichs, M. Gensch, N. Esser, S. Wippermann, W. G. Schmidt, F. Bechstedt, K. Fleischer, and J. F. McGilp, Phys. Rev. Lett. 102, 226805 (2009).

[54] S. Wippermann, W. Schmidt, F. Bechstedt, S. Chandola, K. Hinrichs, M. Gensch, N. Esser, K. Fleischer, and J. McGilp, Phys. Status Solidi C 7, 133 (2010).

[55] W. G. Schmidt, N. Esser, A. M. Frisch, P. Vogt, J. Bernholc, F. Bechstedt, M. Zorn, Th. Hannappel, S. Visbeck, F. Willig, and W. Richter, Phys. Rev. B 61, 16335 (2000).

[56] M. Born, E. Wolf, and A. Bhatia, Principles of Optics: Electromagnetic Theory of Propagation, Interference and Diffraction of Light (Cambridge University Press, Cambridge, UK, 1999).

[57] D. W. Berreman, J. Opt. Soc. Am. 62, 502 (1972).

[58] M. Mansuripur, J. Appl. Phys. 67, 6466 (1990).

[59] R. Verre, M. Modreanu, O. Ualibek, D. Fox, K. Fleischer, C. Smith, H. Zhang, M. Pemble, J. F. McGilp, and I. V. Shvets, Phys. Rev. B 87, 235428 (2013).

[60] W. Plieth and K. Naegele, Surf. Sci. 64, 484 (1977).

[61] T. Tepper, C. Ross, and G. Dionne, IEEE Trans. Magn. 40, 1685 (2004).

[62] K. Fleischer, O. Mauit, and I. V. Shvets, Appl. Phys. Lett. (2014), doi:10.1063/1.4876059. 